\title{
Electrolysis of Water at Atomically Tailored Epitaxial Cobaltite Surfaces
}

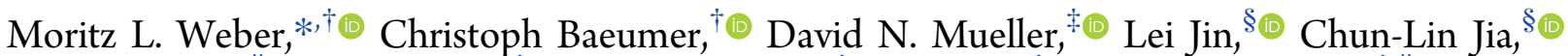 \\ Daniel S. Bick,,$^{\|}$Rainer Waser, ${ }^{\dagger}$ Regina Dittmann, ${ }^{\dagger}$ Ilia Valov, ${ }^{\dagger}$ and Felix Gunkel ${ }^{*}, \dagger, \|_{\odot}$ \\ ${ }^{\dagger}$ Peter Gruenberg Institute 7 and JARA-FIT and ${ }^{\star}$ Peter Gruenberg Institute 6 and JARA-FIT, Forschungszentrum Juelich GmbH, \\ 52425 Juelich, Germany \\ ${ }^{\S}$ Ernst Ruska-Centre for Microscopy and Spectroscopy with Electrons (ER-C), Forschungszentrum Juelich GmbH, 52428 Juelich, \\ Germany \\ "Institute of Electronic Materials (IWE2), RWTH Aachen University, 52074 Aachen, Germany
}

Supporting Information

ABSTRACT: As complex transition-metal oxides of perovskite structures, many cobaltites are active electrocatalysts promoting oxygen evolution reaction (OER) during electrochemical water splitting. To unveil specific structure-activity relationships for electrocatalytic performance, innovative types of catalysts are required to overcome the inherent high complexity of regular powder catalysts, where thin-film technology gained significance in recent years. As we demonstrate, epitaxial $\mathrm{La}_{0.6} \mathrm{Sr}_{0.4} \mathrm{CoO}_{3}$ (LSCO) thin films can be deposited with controlled bulk properties, surface structure, and stoichiometry on orthorhombic (110) $\mathrm{NdGaO}_{3}$ single-crystalline substrates by pulsed-laser deposition, providing ideal model systems for this purpose. The epitaxial thin films are dense and single
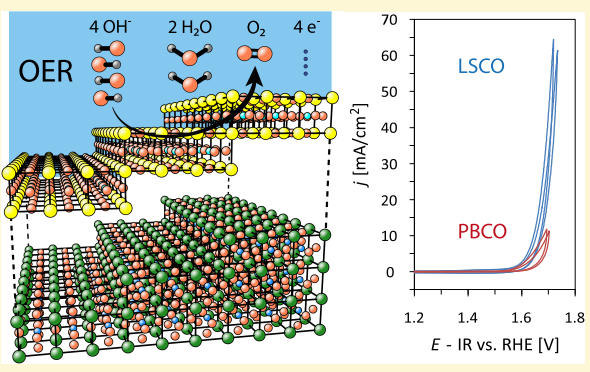
crystalline with sub-nanometer surface roughness and grow well oriented toward the pseudocubic [001] direction. The LSCO thin films show high activity catalyzing the OER and can carry significant current density loads exceeding $100 \mathrm{~mA} / \mathrm{cm}^{2}$. Using these model catalysts, X-ray photoemission spectroscopy reveals the degradation of the material under these dynamic conditions, involving cation leaching and a phase transformation of the oxide. An altered surface stoichiometry as well as cobalt hydroxide formation is observed. Our results show that epitaxial model systems can be operated at large current density loads, allowing a systematic study of catalysts and their degradation under highly dynamic conditions.

\section{INTRODUCTION}

With respect to the steady increase of worldwide energy demands and the ongoing change in perspective regarding conventional power generation, sustainable energy technologies are in focus more than ever. To counter the concomitant intermittency in energy production, efficient energy conversion and storage systems are necessary. ${ }^{1-3}$ Electrochemical water splitting is one promising approach using the incidental excess energies of intermittent energy sources for the production of hydrogen. However, the application of water splitting is limited by its efficiency, in particular due to the sluggish kinetics of the anodic oxygen evolution reaction (OER). ${ }^{4-6}$ The search for novel catalyst materials is driven by the requirement to overcome the need for noble metals, where the great variety of complex oxides with a perovskite structure raised particular

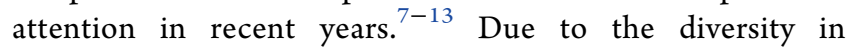
composition and stoichiometry of these oxides, a deeper understanding of the catalytic processes on the atomic level is a fundamental requirement for the rational design of efficient oxide catalysts. Since these atomistic processes during electrocatalysis at active oxide surfaces often remain unclear, research strives to examine these universal descriptors (electronic, structural parameters, etc.), promoting electrocatalytic performance. ${ }^{14-17}$

For classical powder catalysts with highly complex and variable properties, it is often difficult to selectively vary one specific parameter due to their inherent polymorphism and complexity, hampering the investigations of structure-activity relationships. To reduce the complexity of the catalyst system, epitaxial thin films grown by pulsed-laser deposition (PLD) on single-crystalline substrates are well suited for application as catalytic model electrodes as they possess well-defined surfaces, single-crystal orientations, and defined strain states (Figure 1).

Among the perovskite catalysts studied, $\operatorname{Pr}_{0.5} \mathrm{Ba}_{0.5} \mathrm{CoO}_{3}$ (PBCO) and $\mathrm{La}_{0.6} \mathrm{Sr}_{0.4} \mathrm{CoO}_{3}$ (LSCO) received special attention. $8,9,18,19$ These compounds are available in a wide range of cation stoichiometries and can moreover form ordered defect structures potentially promoting catalysis or stability and rendering rich fields of research for physical descriptors. ${ }^{8,9,12}$ In particular, O $2 \mathrm{p}$ band alignment controlled via stoichiometry variations was suggested as a potential

Received: October 30, 2018

Revised: March 12, 2019

Published: March 13, 2019 


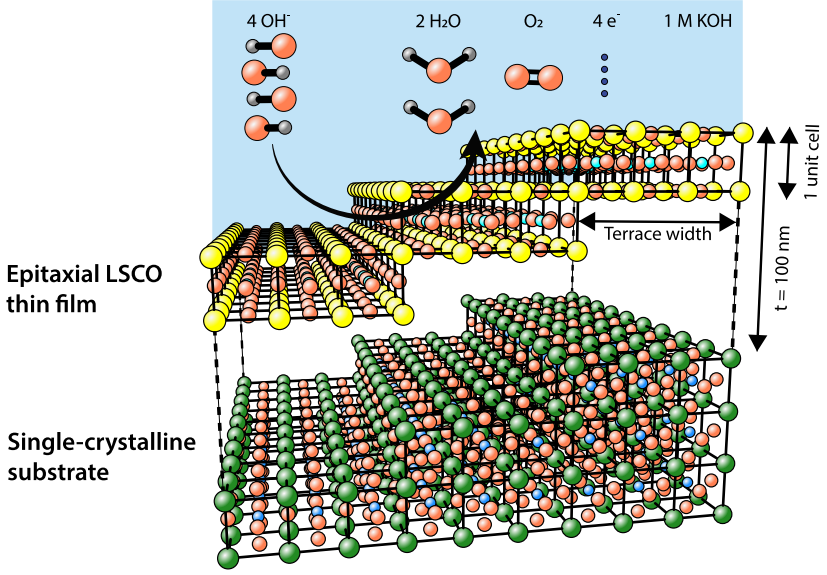

Figure 1. Schematic illustration of the oxygen evolution reaction proceeding in alkaline media at the surface of the active thin film. The $100 \mathrm{~nm}$ thick $\mathrm{La}_{0.6} \mathrm{Sr}_{0.4} \mathrm{CoO}_{3}$ (LSCO) thin film, adapting the surface morphology (terrace structure) of the underlying $\mathrm{NdGaO}_{3}$ (NGO) substrate during the controlled deposition, is forming the catalyst model system.

descriptor for catalytic performance., ${ }^{9,16,18}$ Unlike many conventional oxides however, cobaltites and the late $3 \mathrm{~d}$ transition metals in general deviate from simple ionic behavior. Considerable charge transfer from oxygen anions to cobalt cations has been revealed, ${ }^{20-22}$ adding additional complexity to understanding atomistic processes of surface chemical reactions in these compounds.

Epitaxial thin-film electrodes can be deposited with tailored properties by variation of the growth conditions, for example, regarding their surface orientation, bulk structure, or stoichiometry. ${ }^{8,10,23,24}$ In that way, complex oxide catalyst systems providing specific bulk and surface properties can be studied under maximum controlled catalysis conditions with respect to their electrocatalytic performance. While being welldefined model systems, epitaxial thin films grown by PLD minimize the active surface area. From a technological approach, epitaxial thin films are thus considered unsuitable as they limit the achievable current throughput of the catalyst device (typically to the $\mu \mathrm{A} / \mathrm{cm}^{2}$ or lower $\mathrm{mA} / \mathrm{cm}^{2}$ range). ${ }^{8,10,11,19,23}$ It is often difficult to study the catalyst behavior under large applied current density loads, relevant to the technical community, when using model catalyst systems. Therefore, there exists an essential need in the field to close the apparent gap between model systems aiming at improved understanding of catalytic processes and realistic current density loads applied to technologically relevant catalysts.

In this study, we show that epitaxial LSCO thin films provide a suitable model system for OER catalysis, capable of carrying large current density loads, while at the same time being dense, well oriented, and atomically flat. Epitaxial PBCO, a well-known OER catalyst material, serves as the reference material to evaluate the electrochemical performance of the epitaxial LSCO electrocatalyst. The current density achieved for LSCO is about $1-2$ orders of magnitude higher than the ones typically reported for epitaxial model systems $8,10,11,19,23$ and well comparable to the ones obtained with powder catalysts of the same compounds. ${ }^{9,18}$ As we show, atomically controlled epitaxy of catalyst layers can hence result in equal functionality as conventional powder catalysts, setting model catalyst studies on an equal footing with conventional powder approaches.

\section{EXPERIMENTAL SECTION}

[001]-oriented $\mathrm{La}_{0.6} \mathrm{Sr}_{0.4} \mathrm{CoO}_{3}$ (LSCO) thin films with thicknesses of $100 \mathrm{~nm}$ were grown on one-side-epipolished single-crystalline $\mathrm{NdGaO}_{3}$ (NGO) substrates (CrysTec $\mathrm{GmbH}$, Berlin, Germany) in orthorhombic (110) surface orientation (unless specified, the pseudocubic notation will be used hereafter) by reflection highenergy electron diffraction (RHEED)-controlled PLD. The deposition was performed at an oxygen pressure of $p\left(\mathrm{O}_{2}\right)=0.053 \mathrm{mbar}$, a substrate temperature of $T=650^{\circ} \mathrm{C}$, a repetition rate of $f=5 \mathrm{~Hz}$, and a laser fluence of $2.19 \mathrm{~J} / \mathrm{cm}^{2}$ using a ceramic LSCO target with a target-to-substrate distance of $d=60 \mathrm{~mm}$. The PLD system was operated with a nanosecond KrF-excimer laser (Lambda Physik Lasertechnik, Göttingen, Germany) with a wavelength of $\lambda=248 \mathrm{~nm}$. Surface morphology and roughness were investigated by atomic force microscopy (AFM, Cypher, Oxford Instruments Asylum Research Inc., Santa Barbara). Crystal structure and strain state of the layers were investigated by standard X-ray diffraction (XRD, D8 Advance, Bruker, Massachusetts) and reciprocal space mapping (RSM, PW 3020 X'Pert Diffractometer, PANalytical, The Netherlands). The sample for atomic-resolution scanning transmission electron microscopy (STEM) investigations was prepared by focused ion milling using a dual-beam system (FEI Helios NanoLab400s FIB-SEM). ${ }^{25}$ High-angle annular dark-field (HAADF) imaging was performed using a microscope operated at $200 \mathrm{kV}$, equipped with a high brightness field emission gun, a probe spherical aberration correction system, and a super-X energy-dispersive $\mathrm{X}$-ray spectroscopy system (FEI Titan G2 80-200 ChemiSTEM). ${ }^{26}$ The convergence semiangle for HAADF STEM imaging was approximately $25 \mathrm{mrad}$, and the collection semiangle was 70-200 mrad. For electrical characterization, four-point-probe resistivity and Hall measurements were applied.

Cation stoichiometry of the catalyst layers was investigated before and after electrocatalysis by bulk-sensitive inductively coupled plasma mass spectrometry (ICP-MS) analysis (Agilent 7500ce) and by surface-sensitive X-ray photoemission spectroscopy (XPS) (Phi 5000 VersaProbe, ULVAC Phi, Physical Electronics Inc.). Since ICP-MS requires dissolution of the thin-film material, measurements were performed on two separate samples deposited under identical growth conditions. Nondestructive XPS measurements were performed using the $\mathrm{Al} \mathrm{K} \alpha_{1}$ line $\left(E_{\lambda}=1486.6 \mathrm{eV}\right.$, full width at half-maximum $=0.26$ $\mathrm{eV}$ ) of a monochromized X-ray source. O 1s-, Co 2p-, Sr 3d-, and La $3 \mathrm{~d}$-core-level as well as valence band spectra were analyzed at a photoemission angle of $\theta=15^{\circ}$. For all measurements, the pass energy was kept constant at $E_{0}=29.35 \mathrm{eV}$ for high energy resolution in the fixed analyzer transmission mode. All spectra were aligned to the $\mathrm{C}$ 1s core-level signal.

For electrochemical characterization, i.e., cyclic voltammetry (CV) and electrochemical impedance spectroscopy (EIS), $50 \mathrm{~nm}$ thick Pt contact pads of $8 \mathrm{~mm} \times 0.5 \mathrm{~mm}$ were sputtered on the four edges of the thin films, providing Ohmic contact to the active LSCO thin film. To mount the thin films on the electrode supply, adhesive copper strips were soldered to the platinum contact using indium foil, providing contact to the potentiostat leads (Metrohm Autolab B.V., The Netherlands). To maintain a defined area of the active LSCO surface exposed to the electrolyte $(1 \mathrm{M} \mathrm{KOH})$, an o-ring of $6 \mathrm{~mm}$ inner diameter shields the outer part of the sample, in particular, the platinum contacts, from the electrolyte. Electrochemical characterization was performed at room temperature using a $\mathrm{Hg} / \mathrm{HgO}$ reference electrode (Radiometer Analytical) and a Pt-mesh counter electrode. EIS measurements were carried out for each sample at frequencies between $1 \mathrm{~Hz}$ and $100 \mathrm{kHz}$. During CV, the potential was cycled at the rate of $0.1 \mathrm{~V} / \mathrm{s}$ performing $30 \mathrm{CV}$ cycles.

\section{RESULTS AND DISCUSSION}

The epitaxial LSCO model catalysts were synthesized by PLD in (001) surface orientation on $\mathrm{NdGaO}_{3}$ substrates of (110) orthorhombic surface orientation (corresponding to (001) in pseudocubic notation) having a small lattice mismatch with the 

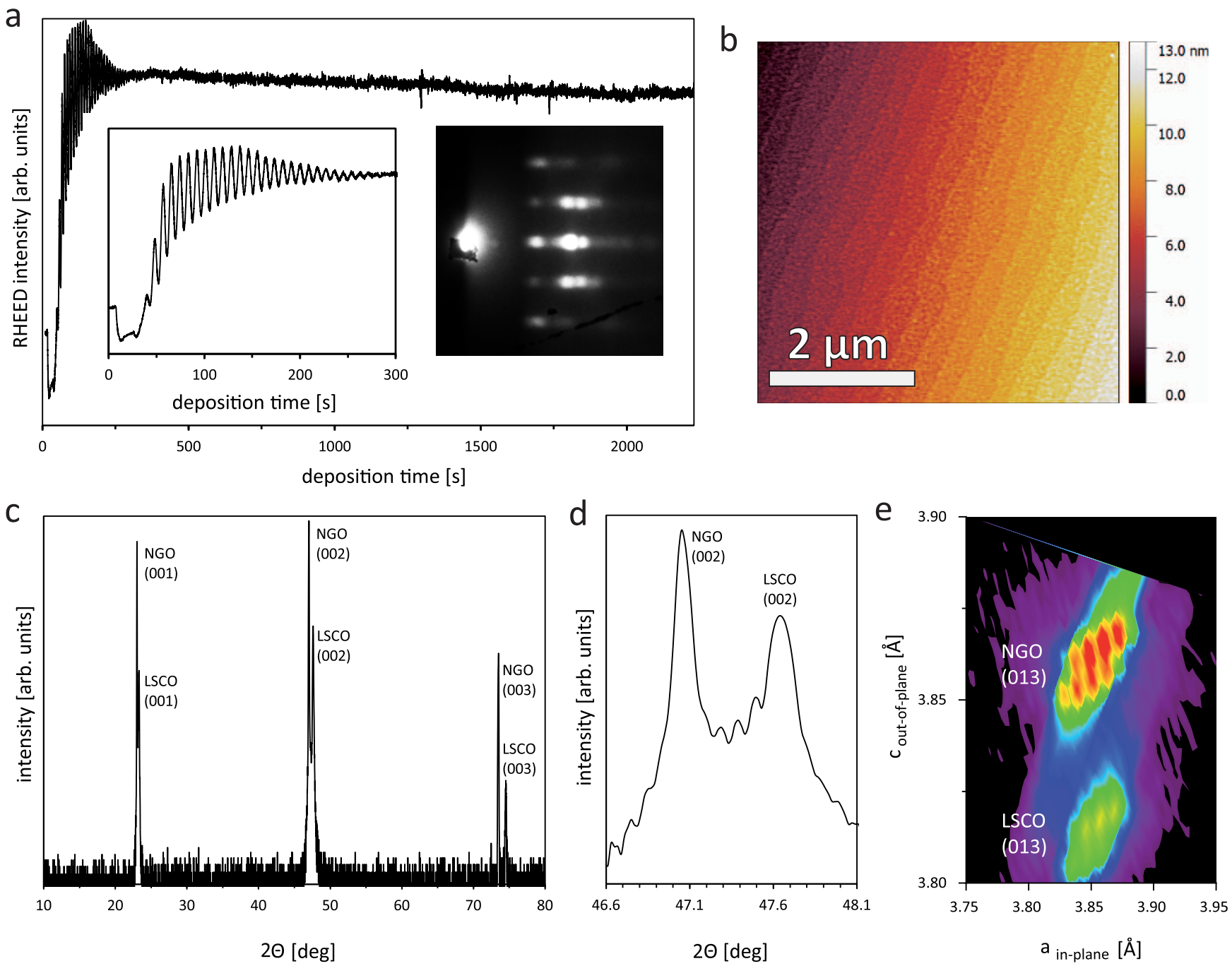

Figure 2. (a) RHEED intensity evolution during PLD growth of LSCO and (inset image, left) a more detailed illustration of the initial growth phase as well as (inset image, right) the corresponding RHEED pattern. (b) Surface morphology of $100 \mathrm{~nm}$ thick epitaxial LSCO thin films (AFM scanning $\left.5 \times 5 \mu \mathrm{m}^{2}\right)$; (c) wide-angle X-ray diffraction analysis; (d) detailed XRD scan in the NGO/LSCO (002) region; and (e) reciprocal space map of the (013) peak.

LSCO target material $(-0.8 \%)$. Hence, only minor tensile strain is induced on the film material. In contrast, LSCO-based thin films reported for use in high-temperature fuel cell concepts $^{27-30}$ are often limited to the choice of yttria-stabilized zirconia single-crystal substrates, serving as a solid oxide electrolyte, but limiting accessible growth modes during PLD due to larger lattice mismatch. Electrolysis of water does not rely on a particular substrate, thus allowing a free choice of perovskite single-crystalline substrates. ${ }^{31}$ The use of orthorhombic (110) $\mathrm{NdGaO}_{3}$ substrates significantly improves epitaxy and allows in situ characterization and control of the fabrication process. For this, we employ reflection high energy electron diffraction (RHEED) during deposition, enabling one to monitor the surface diffraction pattern and surface roughness of the growing film during the fabrication process. The initial growth phase proceeds in a defined layer-by-layer growth as reflected by RHEED intensity oscillations (Figure 2a). Here, each intensity oscillation refers to the growth of a single monolayer of LSCO. A transition of the growth mode is displayed after $\sim 300 \mathrm{~s}$ of deposition time. Attenuation of RHEED intensity oscillations is observed, eventually leading to a constant intensity evolution during the final growth phase, corresponding to a step-flow-like growth mode (Figure 2a). Since both layer-by-layer and step-flow growth modes proceed with a two-dimensional (2D) deposition of the material, LSCO thin films are grown with high accuracy over the entire deposition time. Consistent with the RHEED pattern recorded after the growth of $100 \mathrm{~nm}$ LSCO (inset of Figure 2a), showing a superposition of a $2 \mathrm{D}$ diffraction pattern with minor three-dimensional (3D)-like diffraction domains, AFM imaging revealed close-to atomically flat thin-film surfaces with a clear step terrace structure (Figure $2 b$ ). The terrace step height is one unit cell, whereas the terrace width is typically on the order of a few hundred nanometers. Confirming the dominant 2D growth of the thin films, AFM reveals a subnanometer mean roughness of typically root mean square $<0.7$ $\mathrm{nm}$, where only grainlike structures with sub-unit-cell thickness appear on top of the terrace steps. X-ray diffraction investigations in $2 \theta / \theta$ geometry confirm the epitaxy and the coherent growth of single-phase perovskite LSCO films in [001] orientation (Figure 2c). Finite thickness fringes in the vicinity of the LSCO (002) peaks (Figure 2d) give evidence of the high crystallinity of the thin films. Moreover, reciprocal space mapping, recorded around the (013) reflection of the 
substrate and thin-film material, reveals an adoption in the $\mathrm{NdGaO}_{3}$ in-plane lattice spacing by the LSCO thin film (Figure 2e). Accordingly, the fully strained growth of the thin films is evident.

The as-grown thin films were investigated along the $[100]$ direction by atomic-resolution STEM, confirming fully coherent growth of the LSCO layer and a sharp interface between the thin film and the NGO substrate (Figure 3). The
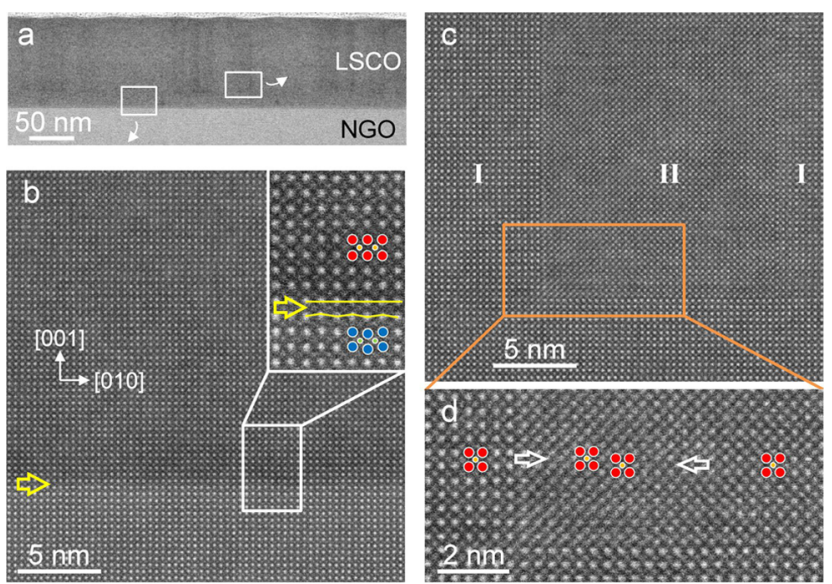

Figure 3. High-resolution STEM analysis for representative LSCO thin-film samples grown on $\mathrm{NdGaO}_{3}$ substrates. In high annular angle dark-field (HAADF) imaging condition, intensity of the image peaks at atomic columns depends on elemental mass so that large $Z$-atomic numbers result in bright contrast. For the given viewing direction, the $\mathrm{NdO}$ atomic columns in the substrate show the brightest intensity and the mixed A-site $\mathrm{SrO} / \mathrm{LaO}$ atomic columns of the $\mathrm{LSCO}$ thin film exhibit less bright intensity, leading to visible contrast between the substrate and thin film across the interface. (a) Overview image indicating a sharp interface between the film and the substrate with occasional extended defects penetrating the thin film perpendicular to the substrate-thin-film interface. (b) Enlarged view of the interface region, confirming epitaxial growth with a coherent interface. (c) Enlarged views of the atomic structure of local defects separating two antiphase domains denoted by I and II. (d) Closeup of an antiphase boundary region where the displacement of the crystal lattice is illustrated.

$Z$-contrast obtained in the HAADF mode yields high image intensities from the A-site atomic columns, i.e., $\mathrm{NdO}$ atomic columns (blue) in the $\mathrm{NGO}$ substrate and $\mathrm{La} / \mathrm{Sr}$ atomic columns (red) in the LSCO thin film, but much weaker intensities from the $\mathrm{GaO}$ (green) and $\mathrm{CoO}$ (orange) columns, as shown in Figure 3b. The overview scan in Figure 3a shows a general morphology of the film with occasional existence of antiphase domains (Figure 3c,d). A closeup at the LSCO/ NGO interface region shows an atomically sharp interface and coherent epitaxy of the film (Figure 3b). The location of the interface (see arrow in Figure $3 \mathrm{~b}$ ) can be traced on the basis of the orthorhombic distortions of the A-site atomic columns in the NGO substrate, as schematically shown in the inset of Figure $3 \mathrm{~b}$. Figure $3 \mathrm{c}$ shows an enlarged view of the LSCO thin film including two antiphase domains I and II. The projected displacement vector is $1 / 2$ [011], as shown in Figure $3 \mathrm{~d}$. Notably, the LSCO thin film shows no detectable evidence for oxygen vacancy ordering, such as observed, for example, in high-temperature-grown $\mathrm{PBCO}$ thin films. ${ }^{8}$

A crucial parameter for electrocatalysts is sufficient electronic conductivity, allowing for charge transfer during
OER. As the NGO substrates used for epitaxial growth are highly insulating, in-plane four-point probe resistivity measurements can be used to deduce the electrical conductivity of the catalyst material only, without any leakage currents or effects of current collecting admixtures during electrical measurements. As shown in Figure 4, we find exceptionally low sheet

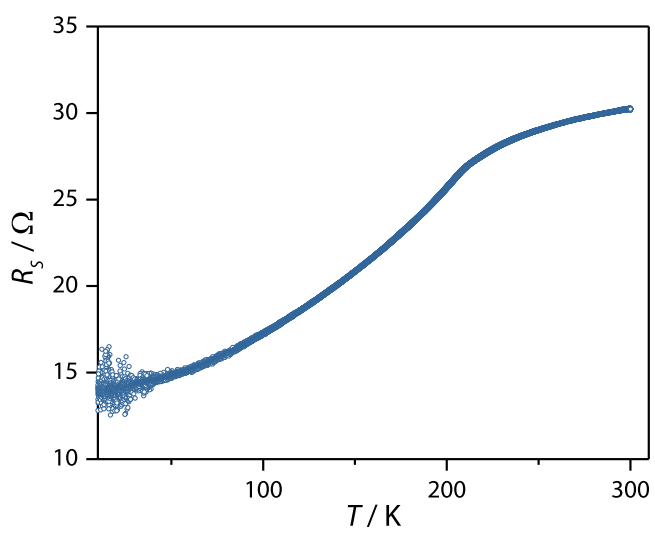

Figure 4. Temperature dependence of sheet resistance determined for $100 \mathrm{~nm}$ thick LSCO thin films, revealing metallic temperature behavior with a relative resistance ratio of 2 .

resistances of $\rho_{\mathrm{S}}=20-30 \Omega / \mathrm{sq}$ at room temperature, corresponding to a conductivity of about $\sigma=3000-5000 \mathrm{~S} /$ $\mathrm{cm}$ determined for various $100 \mathrm{~nm}$ thick LSCO thin films. The high electrical conductivity is favorable for charge transport and for facilitating the transfer of electrons to the active sites of the catalyst during OER. Insufficient electronic conductivity is frequently observed as one limiting factor in electrocatalysis using oxide materials and contributes to an increased effective (over-)potential of the charge transfer reaction. Our LSCO thin films, however, exhibit metallic behavior, evident from the temperature-dependent resistivity data displayed in Figure 4. This behavior is consistent with previous reports indicating metallicity for $x \geq 0.2$ in $\mathrm{La}_{1-x} \mathrm{Sr}_{x} \mathrm{CoO}_{3}$ ceramics ${ }^{20,32}$ as well as with XPS valence band spectroscopy revealing finite density of states at the Fermi level (cf. Figure S1 in the Supporting Information (SI)). Hall measurements performed for the LSCO thin films revealed p-type conduction and a carrier concentration of $p=4.6 \times 10^{21} \mathrm{~cm}^{-3}$ corresponding to 0.26 holes per unit cell as well as a Hall mobility of $2.96 \mathrm{~cm}^{2} /(\mathrm{V} \mathrm{s})$.

Figure 5a shows the two initial voltammetric cycles obtained from epitaxial LSCO thin films, where the total current density is plotted against the applied potential, referenced to the reversible hydrogen electrode (RHE) potential $(\Delta E=+0.924$ $\mathrm{V}$ at $1 \mathrm{M} \mathrm{KOH}, \mathrm{pH}=14)$. The data is shown in comparison to the characteristic initial curves of PBCO, another perovskite complex oxide, recently discussed as a high-performance electrocatalyst for $\mathrm{OER}^{8,9,12}$ and also supplied as a (100) epitaxial thin film. (Note that, for comparison, PBCO thin films were deposited on $\mathrm{SrTiO}_{3}$ single-crystalline substrates, which guarantees a low epitaxial strain state for the $\mathrm{PBCO}$ layer, similar to the one obtained for LSCO grown on $\mathrm{NdGaO}_{3}{ }^{8}$ ) Due to the negligible surface roughness, the active surface provided for electrocatalysis can be considered equal to the geometric surface of the thin films. The cyclovoltammetric data was $i R$-corrected by the total Ohmic series resistance $R$ obtained from electrochemical impedance spectroscopy (EIS), which was carried out for each sample. For this purpose, $R$ was determined by evaluation of the high-frequency intersect of the 
a

.

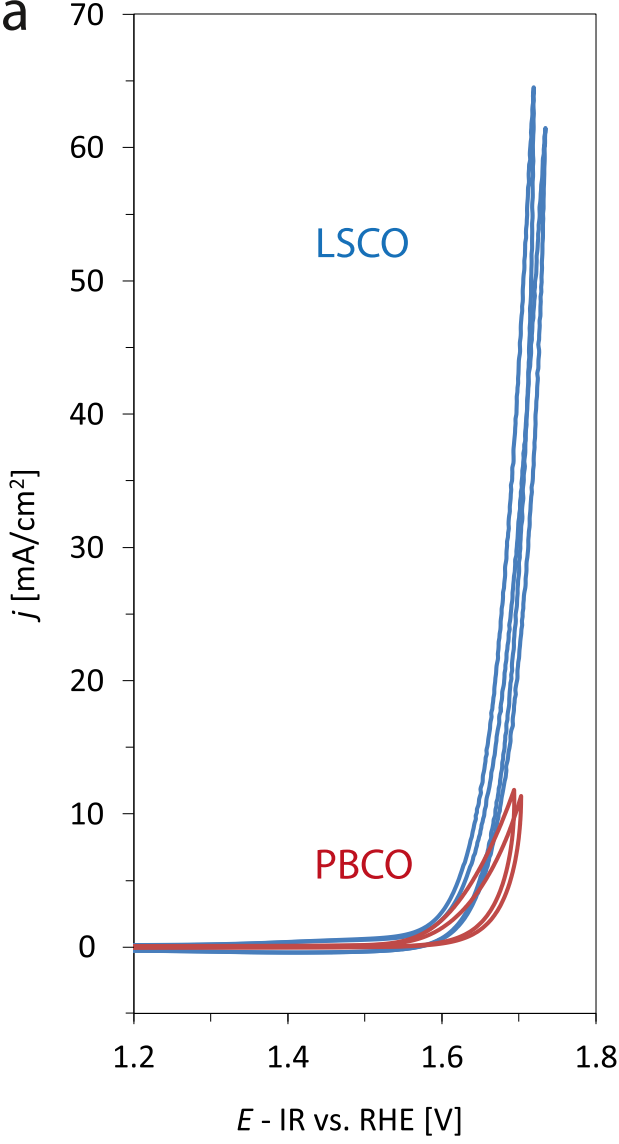

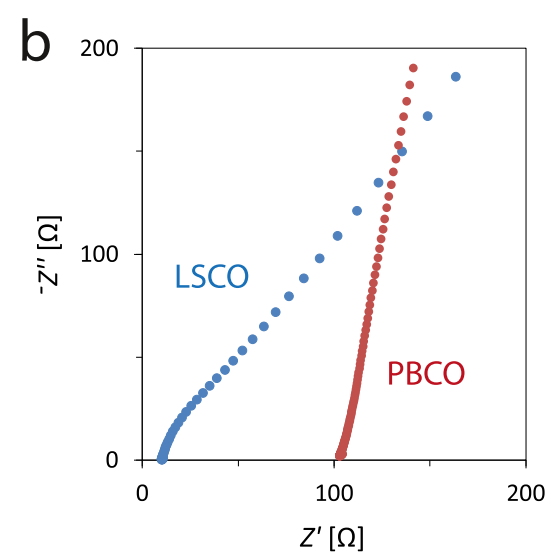

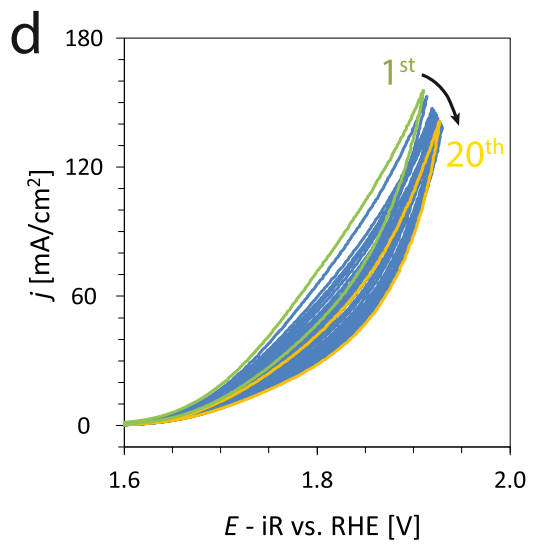

C

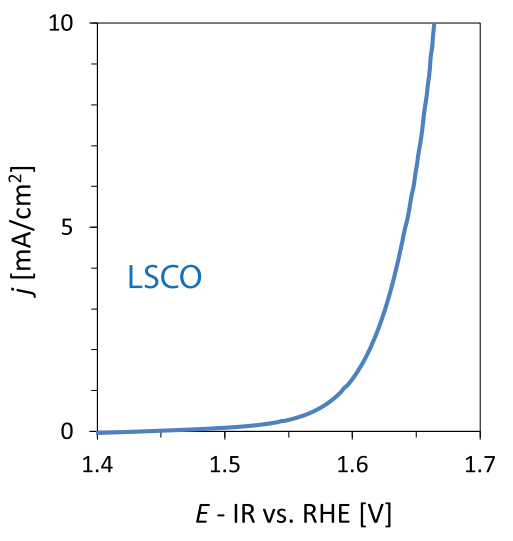

e

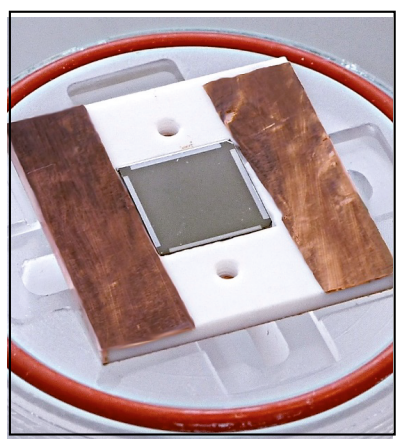

Figure 5. Electrochemical characterization of representative samples in alkaline media $(1 \mathrm{M} \mathrm{KOH})$. Epitaxial thin films of LSCO were grown on $\mathrm{NdGaO}_{3}$, whereas $\mathrm{PBCO}$ thin films were deposited on $\mathrm{SrTiO}_{3}$ substrates. (a) Two initial voltammetric cycles obtained for LSCO/NGO and $\mathrm{PBCO} / \mathrm{STO}$ catalyst systems at $0.1 \mathrm{~V} / \mathrm{s}$. The data was $i R$-corrected based on EIS for each individual sample; the potential was referenced to the RHE potential by $\mathrm{pH}$-dependent correction of the measured potential by the $\mathrm{Hg} / \mathrm{HgO}$ reference potential $(\Delta E=+0.924 \mathrm{~V}$ at $1 \mathrm{M} \mathrm{KOH}, \mathrm{pH}=$ 14). (b) Nyquist plot obtained from epitaxial electrodes by EIS as mounted inside the electrochemical cell. (c) Low overpotential (OER onset) region of the averaged forward and reverse scans of the second CV cycle; (d) $20 \mathrm{CV}$ cycles obtained at high potentials, where the first cycle is denoted in green and the 20th cycle is denoted in yellow. (e) Working electrode setup with the thin film located in the center, before the soldering process.

characteristic curves with the real axis of the Nyquist plot. In general, the resistivity of the thin films can be considered as one major limiting factor for the observed current density during OER (bottleneck for electron transport within the circuit). Consistent with the high conductivity of epitaxial LSCO layers determined by four-point-probe measurements, EIS indicated small resistance values in the range of $R_{\Omega}=4-10$ $\Omega$ (Figure $5 b$ ). These values are comparable to typical Ohmic resistances of the electrolyte itself, indicating that the metallike conductivity of the epitaxial LSCO thin films no longer limits the current density load applied to the epitaxial catalyst. In contrast, $\mathrm{PBCO}$ thin films of similar thickness act as additional series resistance (typically on the order of a few hundreds of ohms (Figure $\left.5 b^{8}\right)$ ). After $i R$ correction, the genuine, inherent catalytic properties of the thin films become accessible. As evident from the cyclovoltagram, current density significantly increases above $\sim 1.6 \mathrm{~V}$ versus RHE (onset potential) for both cobaltite thin films, indicating increasing reaction rates at the surfaces of the (100)-oriented thin films, resulting from oxygen evolution. Yet, electrocatalysis using LSCO thin films, yielding up to several tens of $\mathrm{mA} / \mathrm{cm}^{2}$ in the range of $1.75 \mathrm{~V}$ versus $\mathrm{RHE}$, leads to current densities multiple times larger than PBCO thin films. Based on these high current densities, reflecting the surface interaction between the catalyst and the reactant, the excellent catalytic activity of the epitaxial LSCO films for the OER is evident. Figure 5c highlights the OER onset region at about $1.6 \mathrm{~V}$. In this potential region, the current densities observed for epitaxial LSCO are well comparable to the ones reported for LSCO powder catalysts, ${ }^{18}$ whereas they are a bit smaller than the highest current density values achieved for BSCF, reported to be one of the most active, but also unstable, perovskite catalysts. ${ }^{9,16}$ A log-scale plot is provided in Figure S3. In Figure 5d, 20 consecutive CV cycles up to higher potentials are shown for the LSCO samples. As can be seen, the epitaxial LSCO thin-film catalyst is capable of carrying current density loads even exceeding 100 $\mathrm{mA} / \mathrm{cm}^{2}$ at potentials up to $1.9 \mathrm{~V}$. In contrast, for epitaxial PBCO thin films, hardly any current density above $10 \mathrm{~mA} / \mathrm{cm}^{2}$ can be achieved, which-after $i R$ correction-may be attributed to a slower surface reaction rate on the PBCO surface as compared to the LSCO one. Note, however, that the diminished reaction rate observed in the PBCO case may also be caused by the weaker electrical conductivity and the resulting inhomogeneous potential and current density distribution of the catalyst as compared to the metallic LSCO. Our observation hence does not generally exclude 

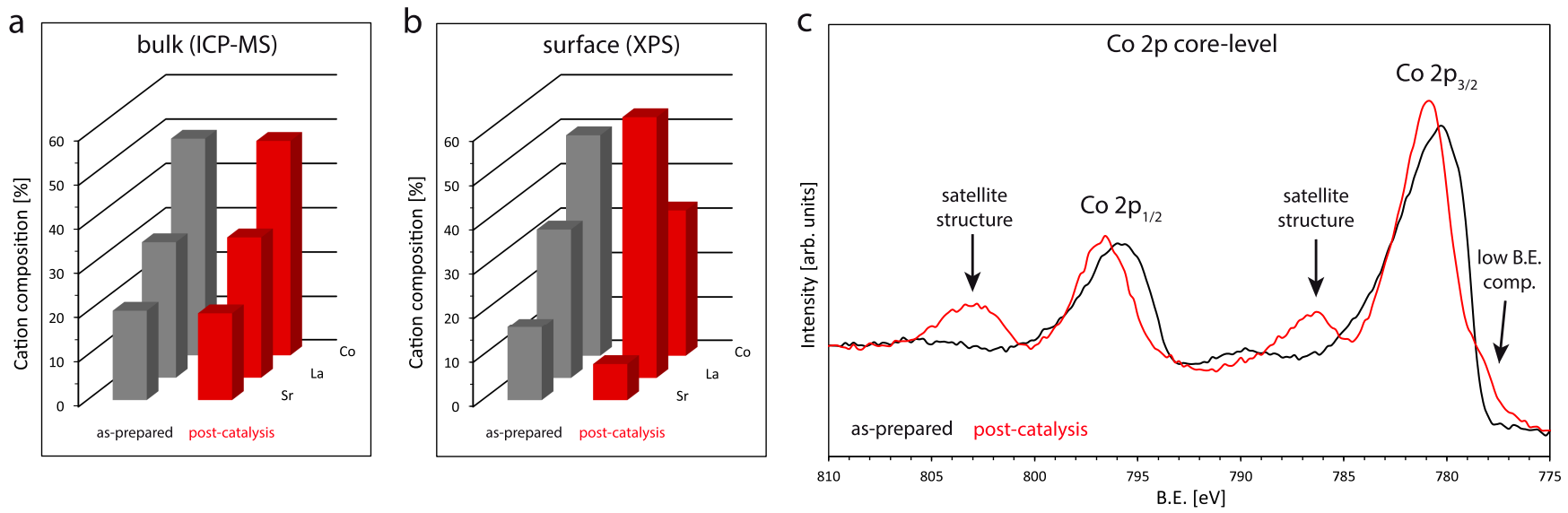

Figure 6. Relative composition of LSCO cations (a) in bulk and (b) in the surface region for the as-deposited state denoted in gray and the postcatalysis state denoted in red. (c) XPS Co $2 \mathrm{p}$ core-level spectra after normalization to the postedge for the as-prepared state shown in black and the postcatalysis state shown in red.

PBCO as a good catalyst. In contrast, it emphasizes the good performance of the epitaxial LSCO layers, which can therefore carry much higher current density loads, well comparable to the ones typically applied to powder catalysts. Thus, also the degradation behavior-likely to scale with applied current density load and potential, but also with the total number of completed surface reactions (i.e., transferred charge)—can be studied under these highly dynamic conditions, not accessible in previously described epitaxial model systems. Moreover, the cyclovoltagram provides basic information about the stability of the catalytic layer. The observed current density and hence OER activity drops slightly over time as visible from decreasing peak values of the consecutive $\mathrm{CV}$ cycles, reflecting the ongoing decrease in catalytic activity due to degradation of the thin films. Note that the $i R$ correction of the $C V$ raw data is solely based on the initial series resistance. Since resistivity typically increases during electrochemical processing (data not shown), both decreasing conductivity and loss in the inherent catalytic activity of the thin-film material might contribute to the decrease in current density as cyclovoltammetry proceeds. Nevertheless, current density remains at high values for all 20 $\mathrm{CV}$ cycles, indicating sufficient stability of the epitaxial catalyst system over the time of electrochemical characterization. It has to be noted that current densities exceeding $100 \mathrm{~mA} / \mathrm{cm}^{2}$ were obtained in the best case. Considerable sample-to-sample variations were observed, typically keeping the current densities on an average of several tens of $\mathrm{mA} / \mathrm{cm}^{2}$ at a potential of $1.75 \mathrm{~V}$ versus RHE. The characteristic overpotential at $10 \mathrm{~mA} / \mathrm{cm}^{2}$ was determined as $\eta_{j_{10}}=396 \mathrm{mV}$ from the forward scan of the second $\mathrm{CV}$ cycle for the best performing sample.

To gain insights into the degradation process of the catalyst material under a large current density load, the NGO/LSCO model system is studied and compared using surface- and bulksensitive techniques carried out in the as-prepared as well as postcatalysis state. For this purpose, XPS provides information about the stoichiometry in the near-surface region and the electronic state of the cobalt transition-metal cations, potentially serving as active sites of the LSCO layer and highly relevant for the catalysis process. $6,9,16,18,33,34$

The composition of cations in the surface region, quantified based on peak areas of XPS core-level spectra as well as bulk composition analyzed by ICP-MS, is shown in Figure 6a,b for the as-prepared and postcatalysis states, respectively (cf. Figure $S 1$ in the Supporting Information for representative core-level spectra). In general, the high accuracy of the thin-film composition in the as-prepared state (gray bars) can be observed. For pristine thin films, bulk-sensitive analysis revealed a $\mathrm{La} / \mathrm{Sr} / \mathrm{Co}$ ratio of $0.63 / 0.41 / 1$, close to the target stoichiometry (Figure 6a). Similarly, only slight deviations from the nominal stoichiometry were determined by XPS for the surface region, originating from a moderate enrichment in lanthanum and depletion from strontium (Figure $6 \mathrm{~b}$ ). The stability of the LSCO layers within the electrolyte (in the absence of any bias) was confirmed by XPS measurements in the soaked state after storing the thin-film catalyst in the electrochemical cell for 45 min (cf. Figure S1e-h), revealing only negligible changes in surface stoichiometry. Furthermore, surface morphology and crystallographic properties of the LSCO layer were not significantly affected by unbiased exposure to the electrolyte (see Figure S2, SI). Cation composition in the postcatalysis state is illustrated by the red bars, where substantial changes in the surface chemistry can be observed, whereas the bulk stoichiometry is not affected by electrochemical processing. Consequently, the degradation of the perovskite phase, based on cation leaching-frequently observed during electrocatalysis at active oxide surfaces and favorable at high potential/high current conditions ${ }^{35}$-is limited to the surface region. While strontium and cobalt cations appear to be preferentially leached out of the perovskite and dissolved in the alkaline media, lanthanum cations show enhanced intensity relative to the other cations during XPS analysis and therefore appear to remain in the surface region. These differences in leaching behavior of cations in LSCO are consistent with the thermodynamic instability reported for Co-based perovskites ${ }^{35-37}$ and the finite solubility of $\mathrm{Sr}(\mathrm{OH})_{2}{ }^{38}$ under catalysis conditions.

In Figure $6 \mathrm{c}$, normalized Co $2 \mathrm{p}$ core-level spectra obtained from thin films in the as-prepared as well as the postcatalysis state are shown, where the spectrum of pristine LSCO is in good agreement with the literature. ${ }^{20,39}$ Overall, the Co corelevel spectrum exhibits a complex structure, making it challenging to provide a satisfying description of the electronic structure by curve fitting. The complex shape of the Co $2 p_{3 / 2}$ and Co $2 \mathrm{p}_{1 / 2}$ doublet with asymmetric main peaks is based on several superimposed signals, resulting from cobalt of different valency as well as multiplet splitting and a significant satellite 

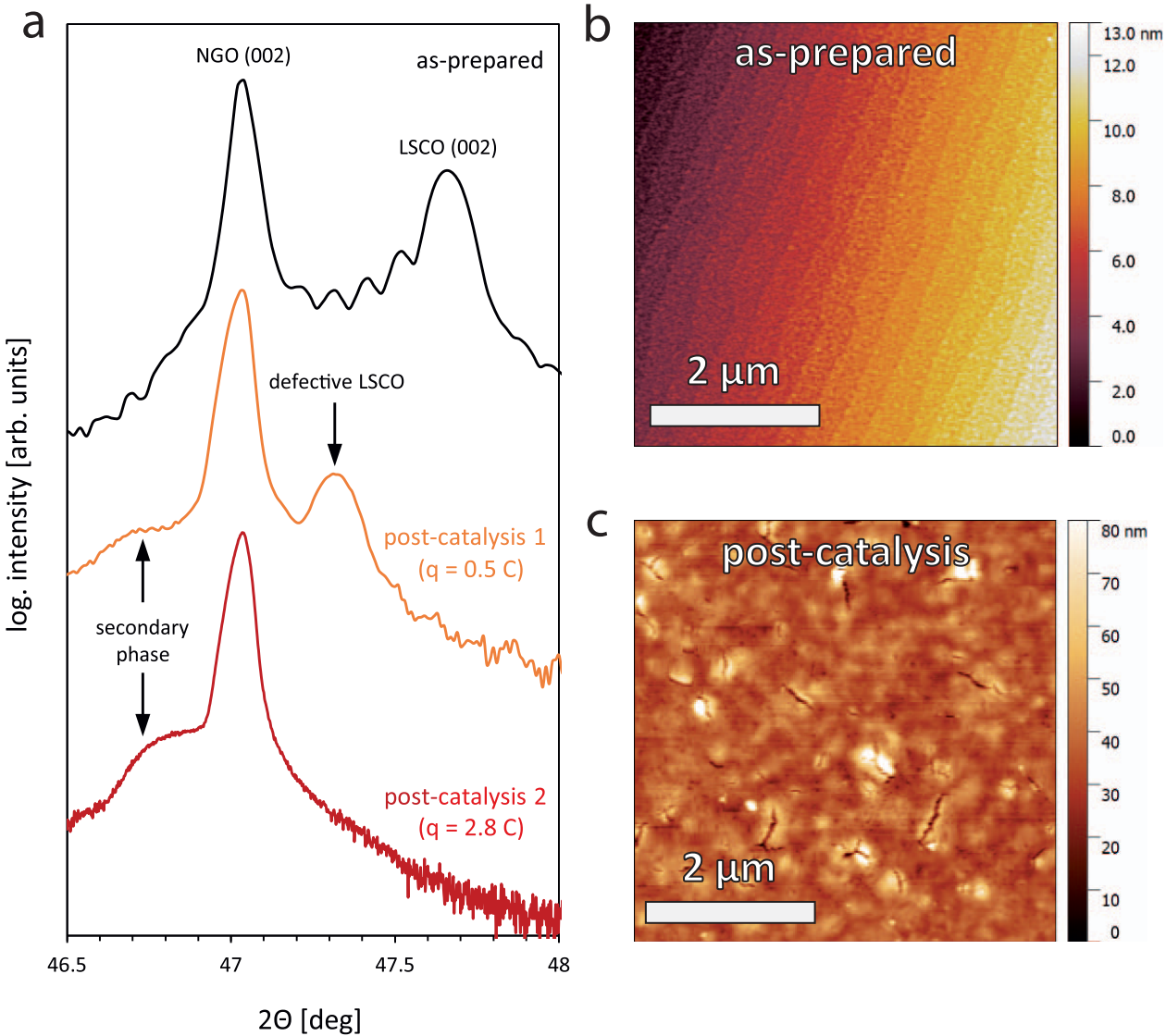

Figure 7. (a) X-ray diffraction analysis in the NGO/LSCO (002) region obtained from thin films in the postcatalysis state. Diffractograms denoted by postcatalysis 1 and postcatalysis 2 were obtained from two different samples after electrochemical operation (after $30 \mathrm{CV}$ cycles) and show considerable changes of the crystallography of epitaxial LSCO thin-film electrodes compared to the as-deposited thin film. Note that the total charge that passed through the layers was $q=0.5 \mathrm{C}$ for the sample "postcatalysis 1 " and $q=2.8 \mathrm{C}$ for the sample "postcatalysis 2 ". Surface morphology of $100 \mathrm{~nm}$ thick epitaxial LSCO thin films (AFM scanning $5 \times 5 \mu \mathrm{m}^{2}$ ); (b) as-prepared and (c) postcatalysis states showing decomposition of the surface due to electrochemical processing.

structure. Further, LSCO is a metallic charge transfer material, with highly covalent character of the $\mathrm{Co}-\mathrm{O}$ bond, ${ }^{20-22}$ leading to additional tailing toward higher binding energies (B.E.s). ${ }^{40-42}$ Therefore, the assignment of formal valence states is not physical in metallic cobaltite perovskites. Because of this complex structure, changes of the electronic structure at the $\mathrm{CoO}_{6}$ manifold responsible for the catalytic activity during catalysis might be subtle in XPS, for example, even for significant hole doping by Sr substitution of LSCO, ${ }^{20,21}$ only small changes in the shape of the respective core-level spectra have been observed. We will therefore limit our discussion to the qualitative level.

Comparing the two cobalt spectra, strong differences can be readily observed in particular with respect to the satellite structure, which is much more pronounced as well as shifted to lower binding energies for spectra obtained from thin films in the postcatalysis state. Considering that these satellite features are generally attributed to divalent cobalt compounds, ${ }^{39,43-45}$ this enhanced satellite signal gives clear evidence of a reduction of the average cobalt valency in the (decomposed) catalytic layer, which must be related with the formation of foreign cobalt compounds. Besides these satellites, the Co $2 p$ spectrum obtained in the postcatalysis state exhibits main peaks of reduced width and enhanced symmetry, indicating a reduction in metallicity of the film material, consistent with the evolution of the valence band spectra (cf. Figure S1 in the Supporting
Information). Furthermore, a shoulder indicating the presence of another cobalt species in the surface region of the thin films appeared at lower binding energies relative to the main peak for the postcatalysis state (Figure 6c, low B.E. component). Referring to the literature, this signal might be assigned to metallic cobalt; ${ }^{42}$ however, it also has been attributed to tetravalent cobalt elsewhere. ${ }^{46}$

These changes in stoichiometry and characteristic core-level features clearly reflect the degradation of the LSCO perovskite, where the formation of altered cobalt phases with a reduced oxidation state of the transition metal associated with the decomposition is evident. Based on reference data provided by Biesinger et al., ${ }^{42}$ this new cobalt phase may be identified as $\mathrm{Co}(\mathrm{OH})_{2}$. This is consistent with the significantly increased hydroxide signal in the $\mathrm{O} 1 \mathrm{~s}$ spectrum after electrocatalysis (cf. Supporting Information). From a thermodynamic perspective however, the formation of $\mathrm{Co}(\mathrm{OH})_{2}$ is surprising under the applied conditions. During electrochemical operation at high potentials and in alkaline media anodic oxidation of cobalt ions and formation of Co species with higher oxidation state, in particular oxyhydroxides would be expected rather than a reduction of the transition metal to the hydroxide form. ${ }^{36,37}$ However, the presence of cobalt oxyhydroxides (i.e., $\mathrm{CoOOH}$ with trivalent cobalt atoms) would not give rise to the observed satellite features in the Co $2 p$ core-level spectrum. ${ }^{44,47}$ 
The strong impact of electrochemical processing on the perovskite phase can also be observed by X-ray diffraction. In Figure 7a, XRD scans of different epitaxial LSCO thin films after potential cycling (30 CVs) are shown, illustrating significant changes in their crystallographic properties compared to the pristine thin films. As can be seen, electrochemical operation results in a shift of the LSCO (002) reflection to lower diffraction angles (Figure $7 \mathrm{a}$, postcatalysis 1). Considering the ongoing leaching of cations from the surface region during catalysis (revealed by our bulkand surface-sensitive studies as described above), this peak shift-indicating an expansion of the thin-film lattice in the $c$ direction-might result from successive accumulation of defects within the crystal lattice. Besides formation of defects, the relaxation of (tensile) strain in the epitaxial thin film might also cause an expansion of the $c$-lattice parameter, contributing to the observed phenomenon. XRD analysis of other thin-film samples in the postcatalysis state even reveals the total decomposition of the crystalline perovskite phase, evident from the full disappearance of the LSCO (002) reflection (Figure $7 \mathrm{a}$, postcatalysis 2 ). Hence, incorporation of defects might be accompanied by either (surface-)amorphization (as reported previously for catalytic oxides ${ }^{13,35,48-51}$ ) or mass loss due to dissolution of the thin-film material based on destabilization of the perovskite phase. Note that the more severe degradation state was obtained as a result of a higher maximum current density and higher applied potential for the sample "postcatalysis 2" in each cycle. Therefore, the total charge that passed through this sample was $q=2.8 \mathrm{C}$, whereas for the sample "postcatalysis 1", which showed an intermediate degradation state, only $q=0.5 \mathrm{C}$ had passed. One has to emphasize however that based on XRD and AFM, we can only show qualitatively that the structural degradation scales with the total amount of transferred charge, whereas a quantitative relation remains elusive.

The time scale of the applied CV experiments is typically a couple of minutes. As shown in Figure S2 (SI), however, the layers remain stable for at least $45 \mathrm{~min}$ in the unbiased case, indicating a strongly accelerated degradation process under OER conditions, implying both significant current density loads and applied potential. Defect formation within the LSCO surface region is consistent with AFM imaging, revealing significant roughening of the initially smooth surface of defined morphology (Figure 7b). Besides the formation of 3D islands of various sizes, deep cracks in the thin-film surface can be observed (Figure 7c).

Furthermore, the formation of a secondary phase can be observed by X-ray diffraction, visible as a broad reflection at lower $2 \theta$ angles relative to the substrate peak (Figure $7 \mathrm{a}$, postcatalyses 1 and 2 ), the nature of which remains to be elucidated in detail. The presence of oriented hydroxides, such as $\mathrm{Co}(\mathrm{OH})_{2}$ and $\mathrm{La}(\mathrm{OH})_{3}$, was considered, but they would be expected to appear at different angular positions.

\section{CONCLUSIONS}

In conclusion, we reported the development of defined, epitaxial LSCO thin-film catalysts for water splitting applications showing high activities for water oxidation in alkaline media. The model catalysts synthesized with subnanometer surface roughness are fully strained and show an atomically defined crystal structure and orientation. The oxidic electrodes show metallic conductivity as well as fast charge transfer at the interface between the electrolyte and the film surface and are capable of carrying large current density during water splitting operation similar to the ones observed in classical powder catalysts. The large accessible current density range makes epitaxial LSCO accessible for degradation studies under highly dynamic operating conditions. Based on XPS, $\mathrm{XRD}$, and AFM analyses, we reveal a degradation process that involves cation leaching from the surface, concomitant with a reduction of cobalt cations toward the formal $2+$ valence state identified as $\mathrm{Co}(\mathrm{OH})_{2}$ species. Upon degradation, an expansion of the LSCO $c$-lattice, the full disappearance of the crystalline catalyst layer as well as the formation of a secondary phase is observed. Using epitaxial thin-film catalysts, appropriate to overcome the inherent polymorphism and complexity of oxide powders, our findings may be specifically attributed to the (001) surface orientation of the LSCO perovskite phase.

The simplicity and the high quality of the crystallographic structure and the surface morphology of the LSCO model catalysts combined with the remarkably high current density range accessible during operation pave ground for further analysis of atomistic processes during OER under realistic operation conditions using catalyst model systems. This includes not only the investigation of structure-activity relationships such as surface orientation and interatomic distances (epitaxial strain) in cobaltite complex oxide catalysts but also local probe analysis and spectroscopy under operando conditions.

\section{ASSOCIATED CONTENT}

\section{S Supporting Information}

The Supporting Information is available free of charge on the ACS Publications website at DOI: 10.1021/acs.chemmater.8b04577.

XPS core-level, survey, and valence band spectra; X-ray diffraction and AFM data obtained after different unbiased exposures to the electrolyte; logarithmic plot illustrating OER activity (PDF)

\section{AUTHOR INFORMATION}

\section{Corresponding Authors}

*E-mail: mo.weber@fz-juelich.de (M.L.W.).

*E-mail: gunkel@iwe.rwth-aachen.de (F.G.).

ORCID

Moritz L. Weber: 0000-0003-1105-2474

Christoph Baeumer: 0000-0003-0008-514X

David N. Mueller: 0000-0002-1062-6985

Lei Jin: 0000-0001-6924-2364

Chun-Lin Jia: 0000-0001-7536-9521

Felix Gunkel: 0000-0003-1178-9986

\section{Notes}

The authors declare no competing financial interest.

\section{ACKNOWLEDGMENTS}

The authors thank Stefan Baumann (Institute of Energy and Climate Research 1, Forschungszentrum Juelich GmbH, 52425 Juelich, Germany) for the preparation of the ceramic LSCO target material. M.L.W. and F.G. thank René Borowski, Paul Meuffels, Volker Nischwitz, Harut Dashtoyan, and Xun Chen for their experimental support. D.N.M. gratefully acknowledges support by the Juelich Joint Redox Lab (JJRL). C.B. has received funding from the European Union's Horizon 2020 
research and innovation program under the Marie SklodowskaCurie grant agreement No. 796142.

\section{REFERENCES}

(1) Chueh, W. C.; Haile, S. M. Electrochemistry of Mixed Oxygen Ion and Electron Conducting Electrodes in Solid Electrolyte Cells. Annu. Rev. Chem. Biomol. Eng. 2012, 3, 313-341.

(2) Götz, M.; Lefebvre, J.; Mörs, F.; Koch, A. M.; Graf, F.; Bajohr, S.; Reimert, R.; Kolb, T. Renewable Power-to-Gas: A technological and economic review. Renewable Energy 2016, 85, 1371-1390.

(3) Varone, A.; Ferrari, M. Power to liquid and power to gas: An option for the German Energiewende. Renewable Sustainable Energy Rev. 2015, 45, 207-218.

(4) Hoare, J. P. The Electrochemistry of Oxygen. J. Electrochem. Soc. 1969, 116, 1168.

(5) Bockris, J. O.; Otagawa, T. The Electrocatalysis of Oxygen Evolution on Perovskites. J. Electrochem. Soc. 1984, 131, 290-302.

(6) Matsumoto, Y.; Sato, E. Electrocatalytic properties of transition metal oxides for oxygen evolution reaction. Mater. Chem. Phys. 1986, 14, 397-426.

(7) Seitz, L. C.; Dickens, C. F.; Nishio, K.; Hikita, Y.; Montoya, J.; Doyle, A.; Kirk, C.; Vojvodic, A.; Hwang, H. Y.; Norskov, J. K.; Jaramillo, T. F. A highly active and stable $\mathrm{IrO}_{x} / \mathrm{SrIrO}_{3}$ catalyst for the oxygen evolution reaction. Science 2016, 353, 1011-1014.

(8) Gunkel, F.; Jin, L.; Mueller, D. N.; Hausner, C.; Bick, D. S.; Jia, C.-L.; Schneller, T.; Valov, I.; Waser, R.; Dittmann, R. Ordering and Phase Control in Epitaxial Double-Perovskite Catalysts for the Oxygen Evolution Reaction. ACS Catal. 2017, 7, 7029-7037.

(9) Grimaud, A.; May, K. J.; Carlton, C. E.; Lee, Y.; Risch, M.; Hong, W. T.; Zhou, J.; Shao-Horn, Y. Double perovskites as a family of highly active catalysts for oxygen evolution in alkaline solution. Nat. Commun. 2013, 4, No. 2439.

(10) Akbashev, A. R.; Zhang, L.; Mefford, J. T.; Park, J.; Butz, B.; Luftman, H.; Chueh, W. C.; Vojvodic, A. Activation of ultrathin $\mathrm{SrTiO}_{3}$ with subsurface $\mathrm{SrRuO}_{3}$ for the oxygen evolution reaction. Energy Environ. Sci. 2018, 11, 1762-1769.

(11) Scholz, J.; Risch, M.; Stoerzinger, K. A.; Wartner, G.; ShaoHorn, Y.; Jooss, C. Rotating Ring - Disk Electrode Study of Oxygen Evolution at a Perovskite Surface: Correlating Activity to Manganese Concentration. J. Phys. Chem. C 2016, 120, 27746-27756.

(12) Bick, D. S.; Griesche, J. D.; Schneller, T.; Staikov, G.; Waser, R.; Valov, I. $\mathrm{Pr}_{x} \mathrm{Ba}_{1-x} \mathrm{CoO}_{3}$ Oxide Electrodes for Oxygen Evolution Reaction in Alkaline Solutions by Chemical Solution Deposition. J. Electrochem. Soc. 2016, 163, F166-F170.

(13) Bick, D. S.; Kindsmueller, A.; Staikov, G.; Gunkel, F.; Mueller, D.; Schneller, T.; Waser, R.; Valov, I. Stability and Degradation of Perovskite Electrocatalysts for Oxygen Evolution Reaction. Electrochim. Acta 2016, 218, 156-162.

(14) Vojvodic, A.; Nørskov, J. K. Optimizing Perovskites for the Water-Splitting Reaction. Science 2011, 334, 1355-1356.

(15) Hong, W. T.; Welsch, R. E.; Shao-Horn, Y. Descriptors of Oxygen-Evolution Activity for Oxides: A Statistical Evaluation. J. Phys. Chem. C 2016, 120, 78-86.

(16) Suntivich, J.; May, K. J.; Gasteiger, H. A.; Goodenough, J. B.; Shao-Horn, Y. A Perovskite Oxide Optimized for Oxygen Evolution Catalysis from Molecular Orbital Principles. Science 2011, 334, 13831385.

(17) Song, F.; Bau, L.; Moysiadou, A.; Lee, S.; Hu, C.; Liardet, L.; $\mathrm{Hu}, \mathrm{X}$. Transition Metal Oxides as Electrocatalysts for the Oxygen Evolution Reaction in Alkaline Solutions: An Application-Inspired Renaissance. J. Am. Chem. Soc. 2018, 140, 7748-7759.

(18) Mefford, J. T.; Rong, X.; Abakumov, A. M.; Hardin, W. G.; Dai, S.; Kolpak, A. M.; Johnston, K. P.; Stevenson, K. J. Water electrolysis on $\mathrm{La}_{1-x} \mathrm{Sr}_{x} \mathrm{CoO}_{3-\delta}$ perovskite electrocatalysts. Nat. Commun. 2016, 7, No. 11053.

(19) Stoerzinger, K. A.; Choi, W. S.; Jeen, H.; Lee, H. N.; ShaoHorn, Y. Role of Strain and Conductivity in Oxygen Electrocatalysis on $\mathrm{LaCoO}_{3}$ Thin Films. J. Phys. Chem. Lett. 2015, 6, 487-492.
(20) Chainani, A.; Mathew, M.; Sarma, D. D. Electron-spectroscopy study of the semiconductor-metal transition in $\mathrm{La}_{1-x} \mathrm{Sr}_{x} \mathrm{CoO}_{3}$. Phys. Rev. B 1992, 46, 9976-9983.

(21) Saitoh, T.; Mizokawa, T.; Fujimori, A.; Abbate, M.; Takeda, Y.; Takano, M. Electronic structure and magnetic states in $\mathrm{La}_{1-x} \mathrm{Sr}_{x} \mathrm{CoO}_{3}$ studied by photoemission and x-ray-absorption spectroscopy. Phys. Rev. B 1997, 56, 1290-1295.

(22) Mueller, D. N.; Machala, M. L.; Bluhm, H.; Chueh, W. C. Redox activity of surface oxygen anions in oxygen-deficient perovskite oxides during electrochemical reactions. Nat. Commun. 2015, 6 , No. 6097.

(23) Petrie, J. R.; Jeen, H.; Barron, S. C.; Meyer, T. L.; Lee, H. N. Enhancing Perovskite Electrocatalysis through Strain Tuning of the Oxygen Deficiency. J. Am. Chem. Soc. 2016, 138, 7252-7255.

(24) Gunkel, F.; Wicklein, S.; Hoffmann-Eifert, S.; Meuffels, P.; Brinks, P.; Huijben, M.; Rijnders, G.; Waser, R.; Dittmann, R. Transport limits in defect-engineered $\mathrm{LaAlO}_{3} / \mathrm{SrTiO}_{3}$ bilayers. Nanoscale 2015, 7, 1013-1022.

(25) Meertens, D.; Kruth, M.; Tillmann, K. FEI Helios NanoLab 400 S FIB-SEM. J. Large-Scale Res. Facil. 2016, 2, No. A60.

(26) Kovács, A.; Schierholz, R.; Tillmann, K. FEI Titan G2 80-200 CREWLEY. J. Large-Scale Res. Facil. 2016, 2, No. A43.

(27) Nenning, A.; Opitz, A. K.; Rameshan, C.; Rameshan, R.; Blume, R.; Hävecker, M.; Knop-Gericke, A.; Rupprechter, G.; Klötzer, B.; Fleig, J. Ambient Pressure XPS Study of Mixed Conducting Perovskite-Type SOFC Cathode and Anode Materials under WellDefined Electrochemical Polarization. J. Phys. Chem. C 2016, 120, 1461-1471.

(28) Kubicek, M.; Rupp, G. M.; Huber, S.; Penn, A.; Opitz, A. K.; Bernardi, J.; Stoeger-Pollach, M.; Hutter, H.; Fleig, J. Cation diffusion in $\mathrm{La}_{0.6} \mathrm{Sr}_{0.4} \mathrm{CoO}_{3-\delta}$ below 800 degrees $\mathrm{C}$ and its relevance for $\mathrm{Sr}$ segregation. Phys. Chem. Chem. Phys. 2014, 16, 2715-2726.

(29) Cai, Z.; Kubicek, M.; Fleig, J.; Yildiz, B. Chemical Heterogeneities on $\mathrm{La}_{0.6} \mathrm{Sr}_{0.4} \mathrm{CoO}_{3-\delta}$ Thin Films - Correlations to Cathode Surface Activity and Stability. Chem. Mater. 2012, 24, 11161127.

(30) Januschewsky, J.; Ahrens, M.; Opitz, A.; Kubel, F.; Fleig, J. Optimized $\mathrm{La}_{0.6} \mathrm{Sr}_{0.4} \mathrm{CoO}_{3-\delta}$ Thin-Film Electrodes with Extremely Fast Oxygen-Reduction Kinetics. Adv. Funct. Mater. 2009, 19, 31513156.

(31) Schlom, D. G.; Chen, L.; Pan, X.; Schmehl, A.; Zurbuchen, M. A. A Thin Film Approach to Engineering Functionality into Oxides. J. Am. Ceram. Soc. 2008, 91, 2429-2454.

(32) Mineshige, A.; Kobune, M.; Fujii, S.; Ogumi, Z.; Inaba, M.; et al. Metal-Insulator Transition and Crystal Structure of $\mathrm{La}_{1-x} \mathrm{Sr}_{x} \mathrm{CoO}_{3}$ as Functions of Sr-Content, Temperature, and Oxygen Partial Pressure. J. Solid State Chem. 1999, 142, 374-381.

(33) Hardin, W. G.; Mefford, J. T.; Slanac, D. A.; Patel, B. B.; Wang, X.; Dai, S.; Zhao, X.; Ruoff, R. S.; Johnston, K. P.; Stevenson, K. J. Tuning the Electrocatalytic Activity of Perovskites through Active Site Variation and Support Interactions. Chem. Mater. 2014, 26, 33683376.

(34) Hardin, W. G.; Slanac, D. A.; Wang, X.; Dai, S.; Johnston, K. P.; Stevenson, K. J. Highly Active, Nonprecious Metal Perovskite Electrocatalysts for Bifunctional Metal-Air Battery Electrodes. I. Phys. Chem. Lett. 2013, 4, 1254-1259.

(35) Han, B.; Risch, M.; Lee, Y.; Ling, C.; Jia, H.; Shao-Horn, Y. Activity and stability trends of perovskite oxides for oxygen evolution catalysis at neutral pH. Phys. Chem. Chem. Phys. 2015, 17, 2257622580.

(36) Bajdich, M.; García-Mota, M.; Vojvodic, A.; Nørskov, J. K.; Bell, A. T. Theoretical Investigation of the Activity of Cobalt Oxides for the Electrochemical Oxidation of Water. J. Am. Chem. Soc. 2013, $135,13521-13530$.

(37) Gerken, J. B.; McAlpin, J. G.; Chen, J. Y. C.; Rigsby, M. L.; Casey, W. H.; Britt, R. D.; Stahl, S. S. Electrochemical Water Oxidation with Cobalt-Based Electrocatalysts from $\mathrm{pH}$ 0-14: The Thermodynamic Basis for Catalyst Structure, Stability, and Activity. J. Am. Chem. Soc. 2011, 133, 14431-14442. 
(38) Haynes, W. M. CRC Handbook of Chemistry and Physics: A Ready-Reference Book of Chemical and Physical Data; CRC Press: Boca Raton, FL, 2013.

(39) Van der Heide, P. A. W. Systematic x-ray photoelectron spectroscopic study of $\mathrm{La}_{1-x} \mathrm{Sr}_{x}$-based perovskite-type oxides. Surf. Interface Anal. 2002, 33, 414-425.

(40) Doniach, S.; Sunjic, M. Many-electron singularity in X-ray photoemission and X-ray line spectra from metals. J. Phys. C: Solid State Phys. 1970, 3, 285-291.

(41) Biesinger, M. C.; Lau, L. W. M.; Gerson, A. R.; Smart, R. S. C. Resolving surface chemical states in XPS analysis of first row transition metals, oxides and hydroxides: $\mathrm{Sc}, \mathrm{Ti}, \mathrm{V}, \mathrm{Cu}$ and $\mathrm{Zn}$. Appl. Surf. Sci. 2010, 257, 887-898.

(42) Biesinger, M. C.; Payne, B. P.; Grosvenor, P.; Lau, L. W. M.; Gerson, A. R.; Smart, R. Resolving surface chemical states in XPS analysis of first row transition metals, oxides and hydroxides: $\mathrm{Cr}, \mathrm{Mn}$, Fe, Co and Ni. Appl. Surf. Sci. 2011, 257, 2717-2730.

(43) Vovk, G.; Chen, X.; Mims, C. In situ XPS studies of perovskite oxide surfaces under electrochemical polarization. J. Phys. Chem. B

2005, 109, 2445-2454.

(44) Mcintyre, N. S.; Cook, M. G. X-Ray Photoelectron Studies on Some Oxides and Hydroxides of Cobalt, Nickel, and Copper. Anal. Chem. 1975, 47, 2208-2213.

(45) Deng, J.; Zhang, L.; Dai, H.; He, H.; Au, C. Single-crystalline $\mathrm{La}_{0.6} \mathrm{Sr}_{0.4} \mathrm{CoO}_{3-\delta}$ nanowires/nanorods derived hydrothermally without the use of a template: Catalysts highly active for toluene complete oxidation. Catal. Lett. 2008, 123, 294-300.

(46) Favaro, M.; Yang, J.; Nappini, S.; Magnano, E.; Toma, F. M.; Crumlin, E. J.; Yano, J.; Sharp, I. D. Understanding the Oxygen Evolution Reaction Mechanism on $\mathrm{CoO}_{x}$ using Operando AmbientPressure X-ray Photoelectron Spectroscopy. J. Am. Chem. Soc. 2017, 139, 8960-8970.

(47) Chen, Z.; Kronawitter, C. X.; Yeh, Y.; Yang, X.; Zhao, P.; Yao, N.; Koel, B. E. Activity of pure and transition metal-modified $\mathrm{CoOOH}$ for the oxygen evolution reaction in an alkaline medium. $J$. Mater. Chem. A 2017, 5, 842-850.

(48) Risch, M.; Grimaud, A.; May, K. J.; Stoerzinger, K. A.; Chen, T. J.; Mansour, A. N.; Shao-Horn, Y. Structural Changes of Cobalt-Based Perovskites upon Water Oxidation Investigated by EXAFS. J. Phys. Chem. C 2013, 117, 8628-8635.

(49) May, K. J.; Carlton, C. E.; Stoerzinger, K. A.; Risch, M.; Suntivich, J.; Lee, Y. L.; Grimaud, A.; Shao-Horn, Y. Influence of Oxygen Evolution during Water Oxidation on the Surface of Perovskite Oxide Catalysts. J. Phys. Chem. Lett. 2012, 3, 3264-3270.

(50) Bick, D. S.; Krebs, T. B.; Kleimaier, D.; Zurhelle, A. F.; Staikov, G.; Waser, R.; Valov, I. Degradation Kinetics during Oxygen Electrocatalysis on Perovskite-Based Surfaces in Alkaline Media. Langmuir 2018, 34, 1347-1352.

(51) Bick, D. S.; Kindsmueller, A.; Cho, D.-Y.; Yousef Mohamed, A.; Bredow, T.; Laufen, H.; Gunkel, F.; Mueller, D. N.; Schneller, T.; Waser, R.; Valov, I. Self-Assembling Oxide Catalyst for Electrochemical Water Splitting 2019, arXiv:1707.03346. arXiv.org e-Print archive. https://arxiv.org/abs/1707.03346. 